hep-th/9510159

McGill-95/50

October, 1995.

\title{
Hawking Radiation and Ultraviolet Regulators
}

\author{
N. Hambli and C.P. Burgess \\ Physics Department, McGill University \\ 3600 University St., Montréal, Québec, CANADA, H3A $2 T 8$.
}

\begin{abstract}
Polchinski has argued that the prediction of Hawking radiation must be independent of the details of unknown high-energy physics because the calculation may be performed using 'nice slices', for which the adiabatic theorem may be used. If this is so, then any calculation using a manifestly covariant — and so slice-independent — ultraviolet regularization must reproduce the standard Hawking result. We investigate the dependence of the Hawking radiation on such a short-distance regulator by calculating it using a Pauli-Villars regularization scheme. We find that the regulator scale, $\Lambda$, only contributes to the Hawking flux by an amount that is exponentially small in the large variable $\Lambda / T_{H} \gg 1$, where $T_{H}$ is the Hawking temperature; in agreement with Polchinski's arguments. We also solve a technical puzzle concerning the relation between the short-distance singularities of the propagator and the Hawking effect.
\end{abstract}

\section{Introduction}

The prediction that very massive stars must end their days as black holes has by now become deeply ingrained into common astrophysical lore. Our belief in this result rests in no small part on the continued success with which General Relativity accounts for observations, both within the solar system and beyond. 
Part of the progress of the last twenty years has been the integration of this success into the broader body of laws which describe the other known, nongravitational, interactions. It is now understood that, in spite of the notorious obstacles to constructing a full quantum theory of gravity, semiclassical General Relativity can be interpreted as a controllable lowenergy approximation to whatever unknown physics might ultimately describe nature on the very shortest of length scales. In this sense, General Relativity joins other venerable, but nonrenormalizable, low-energy effective theories [1], and semiclassical calculations are justified for observables that vary on distance scales that are long compared to the Planck length.

Perhaps the biggest surprise to emerge from the study of semiclassical quantum physics in the presence of macroscopic gravitational fields is Hawking's discovery [2], that black holes constantly radiate subatomic particles. These particles dominantly emerge far from the hole with energies that are of order the Hawking temperature: $E \simeq T_{H} \equiv\left(4 \pi r_{s}\right)^{-1}$, where $^{1} r_{s}=2 G M$ is the Schwarzschild radius for a black hole of mass $M$. Provided that the hole is sufficiently massive $-G M^{2} \gg 1$ (or, in cgs units, $M \gg 22 \mu g$ ) — the radiation is a long-wavelength effect, and so one expects its semiclassical description to be justified.

It therefore comes as something of a surprise, as was originally emphasized in [3], and more recently in [4] and [5], to find that the standard derivations of the Hawking effect (in four dimensions) make reference in one way or another to physics at extremely short distances. This is true both of Hawking's original derivation, as well as of more modern alternatives [6].

The short distances arise because the Hawking radiation is defined to be the flux which emerges at very late times, well after all of the transients associated with the stellar collapse itself have passed. However, in the usual derivations the radiation which emerges at the Hawking temperature at such late times is strongly redshifted as it climbs out of the black hole's gravitational well. Alternatively, in the formalism set up in Ref. [6], the outgoing flux is derived from the short-distance form for the radiated particle's two-point (Hadamard) function (see below for details) as its position arguments, $x$ and $x^{\prime}$, approach one another and the event horizon.

Polchinski [7], on the other hand, has argued persuasively that, in spite of these appearances, Hawking radiation is nevertheless a robust feature of the long-distance theory. His arguments use the ability, in principle, to perform one's calculations using only 'nice slices' for which curvatures are everywhere small, and for which the adiabatic theorem ensures all high-frequency modes must be in their ground state.

1 We use fundamental units, $\hbar=c=k_{B}=1$, throughout. 
Our purpose here is to present evidence supporting Polchinski's arguments using an explicit calculation of the Hawking radiation in a simple model. We perform the following consistency check on these arguments: if the existence of nice slices guarantees that the Hawking flux is independent of the details of short-distance physics, then any reasonable manifestly-covariant — and so slice-independent — ultraviolet regularization must also not affect this flux. We test this by computing the Hawking radiation using a minimallycoupled massive scalar field in the presence of a Schwarzschild black hole, using a PauliVillars ultraviolet regularization. We are able to implement this regularization by suitably adapting the methods of Fredenhagen and Haag Ref. [6]. We find that all of the cutoff dependence vanishes exponentially in the limit $\Lambda \gg T_{H}$, in agreement with Polchinski's arguments.

The details of this calculation are described in Section (2). In Section (3), we resolve an apparent paradox concerning the relation between the Hawking radiation and the absence of short-distance singularities of the two-point function in the regulated theory. Our conclusions are summarized in Section (4).

\section{A Regulated Example}

In this section we compute the dependence of the Hawking flux on the short-distance regulator.

We take as our observable the outgoing energy flux per unit time, $\mathcal{F} \equiv-\left\langle T_{t}{ }^{r}\right\rangle$, as seen at very late times and at a very large distance from the black hole, with the average taken in the state which corresponds to the vacuum at very early times before the black hole has formed. $t$ and $r$ here represent the usual Schwarzschild coordinates, in terms of which $d s^{2}=-\left(1-r_{s} / r\right) d t^{2}+\left(1-r_{s} / r\right)^{-1} d r^{2}+r^{2}\left(d \theta^{2}+\sin ^{2} \theta d \phi^{2}\right) . \mathcal{F}$ is related to the total black hole luminosity by $L_{H}=\int \mathcal{F} r^{2} \sin \theta d \theta d \phi$.

For a minimally-coupled scalar field the stress tensor is quadratic in the field operator, and so its expectation may be expressed in terms of the coincidence limit of the Hadamard two-point function: $G\left(x, x^{\prime}\right) \equiv \frac{1}{2}\left\langle\varphi(x) \varphi\left(x^{\prime}\right)+\varphi\left(x^{\prime}\right) \varphi(x)\right\rangle$. In our case:

$$
\begin{aligned}
\mathcal{F} & \equiv-\left\langle T_{t}^{r}\right\rangle=-\left\langle T_{t r^{\star}}\right\rangle \\
& =-\frac{1}{2} \lim _{x^{\prime} \rightarrow x}\left(\frac{\partial}{\partial t^{\prime}} \frac{\partial}{\partial r^{\star}}+\frac{\partial}{\partial r^{\star \prime}} \frac{\partial}{\partial t}\right) G\left(x, x^{\prime}\right),
\end{aligned}
$$

where $r^{\star}$ is the 'tortoise' coordinate: $r^{\star} \equiv r+r_{s} \ln \left[\left(r / r_{s}\right)-1\right]$. The problem reduces to the calculation of $G\left(x, x^{\prime}\right)$. 


\section{1) The Regulator}

Since $G\left(x, x^{\prime}\right)$ is singular as $x^{\prime} \rightarrow x$, the components of the stress tensor usually diverge, and so must be regularized and renormalized. (Off-diagonal components are typically finite in Schwarzschild, however.) We choose to perform this regularization à la Pauli-Villars - i.e. by introducing additional fields, $\varphi_{i}(x)$, some with the 'wrong'-sign kinetic energies, in such a way as to ensure the finiteness of the coincidence limit of

$$
G_{\mathrm{reg}}\left(x, x^{\prime}\right)=\sum_{i} \epsilon_{i} G_{i}\left(x, x^{\prime}\right)
$$

where $\epsilon_{i}= \pm$ keeps track of the sign of the corresponding field's kinetic energy. We should also point out that the sum in eq. (2) includes the contribution from the physical field of mass $m$ whose $\epsilon=+$. Our purpose is ultimately to determine how $\mathcal{F}$ depends on the masses of the regularization fields, $M_{i}$, in the limit that $M_{i} \sim \Lambda \gg T_{H} \gtrsim m$, where $\Lambda$ is the inverse of a covariantly-defined cutoff length (see below), and $m$ is the mass of the original scalar field.

The properties of the regulator fields that are required may be directly calculated from the known divergence structure for minimally-coupled free scalar fields propagating through macroscopic background fields. For a scalar field of mass, $m$, there are three independent types of divergences, which are known to be proportional to the following three coefficients [8]: ${ }^{2}$

$$
\begin{aligned}
& C_{0} \equiv m^{4}\left[a_{0}\right]=m^{4}, \\
& C_{1} \equiv m^{2}\left[a_{1}\right]=-\frac{m^{2}}{6} R, \quad \text { and } \\
& C_{2} \equiv\left[a_{2}\right]=\frac{1}{180} R_{\mu \nu \lambda \rho} R^{\mu \nu \lambda \rho}-\frac{1}{180} R_{\mu \nu} R^{\mu \nu}-\frac{1}{30} \square R+\frac{1}{72} R^{2} .
\end{aligned}
$$

Cancellation of all of these short-distance singularities amongst the Pauli-Villars fields is therefore equivalent to the following conditions:

$$
\begin{aligned}
1+\sum_{i} \epsilon_{i} & =0 \\
m^{2}+\sum_{i} \epsilon_{i} M_{i}^{2} & =0 \\
m^{4}+\sum_{i} \epsilon_{i} M_{i}^{4} & =0 .
\end{aligned}
$$

\footnotetext{
2 These expressions use the conventions of Ref. [9].
} 
As is easily verified, a solution to these equations is given by: $\epsilon_{1}=\epsilon_{2}=+, M_{1}^{2}=M_{2}^{2}=$ $3 \Lambda^{2}+m^{2} ; \epsilon_{3}=\epsilon_{4}=-, M_{3}^{2}=M_{4}^{2}=\Lambda^{2}+m^{2} ;$ and $\epsilon_{5}=-, M_{5}^{2}=4 \Lambda^{2}+m^{2}$.

\section{2) Computing the Hawking Flux}

We may now use $G_{\text {reg }}\left(x, x^{\prime}\right)$ to compute the $\Lambda$-dependence of the Hawking flux. We do so by adapting the arguments of Ref. [6] to our example, since this formulation of the calculation is easily applied to massive fields.

Starting from the definition of $G_{\mathrm{reg}}\left(x, x^{\prime}\right)$, and eq. (1), we see that the Hawking flux may be simply written as $\mathcal{F}=\sum_{i} \epsilon_{i} \mathcal{F}_{i}$, where $\mathcal{F}_{i}$ is the Hawking flux due to a minimallycoupled scalar field of mass $M_{i}$. A straightforward application of the techniques of Ref. [6] gives the usual result:

$$
\mathcal{F}_{i}\left(M_{i}\right)=\frac{1}{4 \pi^{2} r^{2}} \sum_{\ell m}\left|Y_{\ell m}(\theta, \phi)\right|^{2} \int_{M_{i}}^{\infty} d \omega\left|\mathcal{T}_{\ell}\left(\omega, M_{i}\right)\right|^{2} \frac{\omega}{e^{\omega / T_{H}}-1}
$$

In this expression, $(r, \theta, \phi)$ are the Schwarzschild coordinates for the point at which $\mathcal{F}_{i}$ is computed, and $Y_{\ell m}$ are the usual spherical harmonics. $\left|\mathcal{T}_{\ell}\left(\omega, M_{i}\right)\right|^{2} \leq 1$ is the probability that an outgoing particle of mass $M_{i}$ and energy $\omega$ (as seen by the stationary observers at infinity) is transmitted from the event horizon $\left(r^{\star} \rightarrow-\infty\right)$ out to infinity, rather than being scattered back to the horizon by the black hole's gravitational field.

Since the regulator fields all satisfy $M_{i} \gg T_{H}$, it suffices to use the asymptotic form for the flux in this limit. In this limit the frequency integral may be bounded from above:

$$
\begin{aligned}
\int_{M_{i}}^{\infty} d \omega\left|\mathcal{T}_{\ell}\left(\omega, M_{i}\right)\right|^{2} \frac{\omega}{e^{\omega / T_{H}}-1} & \leq 2 \int_{M_{i}}^{\infty} d \omega \omega e^{-\omega / T_{H}} \\
& \leq 2 T_{H}^{2}\left(1+\frac{M_{i}}{T_{H}}\right) e^{-M_{i} / T_{H}}
\end{aligned}
$$

We see that, for $\mathcal{F}_{i}$, every term in the sum over $\ell$ is exponentially small in $\Lambda / T_{H}$. Of course, this is just what would be expected for a thermal radiation spectrum.

One might worry that, although each term in the sum over $\ell$ is exponentially small, it may be that the series sums to a result which is not exponentially suppressed. This does not happen, however, because a much stronger bound is possible for $\left|\mathcal{T}_{\ell}\right|^{2}$ when $\ell$ becomes sufficiently large. The better bound arises because for large angular momenta the transmission probability, $\left|\mathcal{T}_{\ell}\left(\omega, M_{i}\right)\right|^{2}$ goes to zero. This can most easily be seen by 
recasting the scattering problem in terms of the quantum mechanics of a single particle moving in the presence of an effective 'potential':

$$
\begin{aligned}
V_{\mathrm{eff}} & \equiv M_{i}^{2}\left(1-\frac{r_{s}}{r}\right)\left(1+\frac{\ell(\ell+1)}{M_{i}^{2} r^{2}}+\frac{r_{s}}{M_{i}^{2} r^{3}}\right) \\
& \approx M_{i}^{2}\left(1-\frac{r_{s}}{r}\right)\left(1+\frac{\ell^{2}}{M_{i}^{2} r^{2}}\right) .
\end{aligned}
$$

This last, approximate, form has been simplified using $\ell \gg 1$ and $M_{i} r_{s} \gg 1$. Classical evolution in this potential simply predicts $\left|\mathcal{T}_{\ell}\right|^{2}=1$ when $\omega$ lies above the potential for all $r$, and $\left|\mathcal{T}_{\ell}\right|^{2}=0$ when $\omega$ is below the barrier for some $r$. For the above potential, however, there is no barrier at all to escape for $\ell \leq L \equiv \sqrt{3} M_{i} r_{s}$, since only for these $\ell$ 's can the centrifugal contribution dominate the gravitational attraction. For $\ell>L$, on the

other hand, $V_{\text {eff }}$ has a maximum for $r=r_{\max } \gtrsim r_{s}$ that can reflect a potentially outgoing particle, and so transmission is forbidden for $\omega<V_{\max }$. But since the height of the barrier, $V_{\max } \sim \ell / M_{i} r_{s}$, grows for large $\ell$, reflection eventually becomes inevitable for sufficiently large $\ell$. Physically, particles with large $\ell$, but fixed $\omega$, are not sufficiently radially directed to escape to infinity once they try to climb out of the black hole's gravitational well. As a result the sum over $\ell$ that appears in $\mathcal{F}_{i}$ is eventually cut off for sufficiently large $\ell$.

We conclude, then, that at least for this regularization, the contribution of very-shortdistance physics, at distances $\sim 1 / \Lambda$, to the Hawking flux is exponentially suppressed by the large ratio $\Lambda / T_{H}$.

\section{Hawking Radiation and the Absence of Singularities}

The result of the last Section raises another question. We have computed the Hawking radiation in a regulated theory having a completely smooth two-point function. But it is also straightforward to show, by trivially extending the arguments of Ref. [6] to massive fields, that a coincidence limit of the form $G\left(x, x^{\prime}\right) \sim 1 /\left[4 \pi^{2} \sigma\left(x, x^{\prime}\right)\right]+$ (less singular) where $\sigma\left(x, x^{\prime}\right)$ denotes the proper separation between the points $x$ and $x^{\prime}$ - is required near $r=r_{s}$ in order to produce the Hawking radiation. That is, in the approach of Ref. [6] the Hawking flux is completely determined by the coefficient of this $1 / \sigma$ singularity of the two-point function, $G\left(x, x^{\prime}\right)$, when the coincidence limit is taken near the black hole event horizon. The question therefore is: How can a nonzero flux be obtained using a regularized propagator which is smooth in the coincidence limit? The present Section is devoted to the resolution of this apparent contradiction.

The starting point for Ref. [6]'s analysis is the observation that eq. (1) allows us to compute the Hawking flux at a point $(T, \mathcal{R}, \Theta, \Phi)$, at large distances from the black hole 
and at late times, given knowledge of $G\left(x, x^{\prime}\right)$ in the neighbourhood of this point. In ref. [6] the two-point function at large distances from the black hole, $G\left(X_{1}, X_{2}\right)$, is related to its values on an earlier spacelike hypersurface using the surface independence of the Klein-Gordon inner product:

$$
(f, g)=\int_{\Sigma} f * \stackrel{\leftrightarrow}{\partial}_{\mu} g d \Sigma^{\mu}
$$

provided that the functions $f$ and $g$ satisfy the Klein-Gordon equation. This leads to the following expression:

$$
G\left(X_{1}, X_{2}\right)=\int_{\Sigma_{\tau}} \int_{\Sigma_{\tau}} d \Sigma_{1}^{\mu} d \Sigma_{2}^{\nu} G\left(x_{1}, x_{2}\right) \stackrel{\leftrightarrow}{\partial}_{1 \mu} \stackrel{\leftrightarrow}{\partial}_{2 \nu} f\left(x_{1}\right) f^{*}\left(x_{2}\right)
$$

where both integrals are taken over the same timelike surface, $\Sigma_{\tau}$, which we may take to be a surface of constant $\tau=t+r^{\star}-r$. The measure for such a surface is $d \Sigma^{\mu}=$ $n^{\mu} r^{2} \sin ^{2} \theta d r d \theta d \phi$, with $n^{\mu}$ the unit normal to $\Sigma_{\tau}$. Explicitly,

$$
n \cdot \partial=\left(1+\frac{r_{s}}{r}\right) \frac{\partial}{\partial \tau}-\frac{r_{s} \partial}{r \partial r}
$$

The function $f(x)$, which appears in Eq. (9) is the particular solution to the Klein-Gordon equation which satisfies the following 'initial' conditions, which we choose to specify on a late-time constant- $t$ surface which contains the point $X=(T, \mathcal{R}, \Theta, \Phi)$ at which the Hawking flux is to be measured

$$
\begin{aligned}
\left.f(x)\right|_{t=T} & =0 \\
\left.\partial_{t} f(x)\right|_{t=T} & =\delta^{3}(\vec{x}-\vec{X}) .
\end{aligned}
$$

The vector symbol here denotes the three coordinates which specify a point on the surface $t=T$.

The Fredenhagen and Haag derivation [6] crucially relies on this surface-independence of the Klein-Gordon inner product, $(f, g)=\int_{\Sigma} f^{*} \stackrel{\leftrightarrow}{\partial}_{\mu} g d \Sigma^{\mu}$, when the functions $f$ and $g$ satisfy the Klein-Gordon equation. In eq. (9), this is applied in particular to the two-point function, $G\left(x, x^{\prime}\right)$. The resolution of the apparent paradox therefore relies on the fact that a regulated propagator like $G_{\mathrm{reg}}\left(x, x^{\prime}\right)$ does not satisfy the Klein-Gordon equation, but rather satisfies a more complicated higher-derivative equation of motion. The conserved inner 
product for this equation of motion also involves higher-derivative corrections, and these corrections are what generate the Hawking flux from a nonsingular two-point function.

We next illustrate this argument with an explicit calculation. Rather than dealing with the cumbersome details of the five regulator fields that are used in the text, for clarity of presentation we instead present an example which uses just one regulator field. Consider, therefore, the following two-point function:

$$
\hat{G}\left(x, x^{\prime}\right) \equiv G_{m^{2}}\left(x, x^{\prime}\right)-G_{M^{2}}\left(x, x^{\prime}\right),
$$

where $G_{m^{2}}\left(x, x^{\prime}\right)$ and $G_{M^{2}}\left(x, x^{\prime}\right)$ respectively denote the two-point functions for a free scalar fields of mass $m$ and $M \gg m$. Comparing to the short-distance expansion of Ref. [8], shows that the coincidence limit of $\hat{G}\left(x, x^{\prime}\right)$ is at worst $\sim \log \sigma\left(x, x^{\prime}\right)$, for Schwarzschild spacetime. Even though this is less singular than $1 / \sigma\left(x, x^{\prime}\right)$, our goal here is to show that $\hat{G}\left(x, x^{\prime}\right)$ nevertheless produces a nonzero Hawking flux.

In order to apply the methods of Ref. [6], we must first find what equation of motion $\hat{G}\left(x, x^{\prime}\right)$ satisfies, and then construct the corresponding conserved 'inner product' for this equation. As is simple to check, the equation of motion is:

$$
\frac{1}{M^{2}-m^{2}}\left(\square-m^{2}\right)\left(\square-M^{2}\right) \hat{G}\left(x, x^{\prime}\right)=0
$$

The conserved 'inner product' for two solutions, $f$ and $g$, of this equation then is:

$$
\begin{aligned}
{[f, g]=- } & \frac{M_{+}^{2}}{M_{-}^{2}} \int_{\Sigma} d \Sigma^{\mu} f^{*} \stackrel{\leftrightarrow}{\partial}_{\mu} g \\
& +\frac{1}{M_{-}^{2}} \int_{\Sigma} d \Sigma^{\mu} f^{*} \stackrel{\leftrightarrow}{\partial}_{\mu} \square g+\frac{1}{M_{-}^{2}} \int_{\Sigma} d \Sigma^{\mu}\left(\square f^{*}\right) \stackrel{\leftrightarrow}{\partial}_{\mu} g
\end{aligned}
$$

where $M_{ \pm}^{2} \equiv M^{2} \pm m^{2}$, and $\Sigma$ is a spacelike surface. Clearly this expression approaches the usual Klein-Gordon one in the limit $M \rightarrow \infty$.

Using this expression to write the analogue of eq. (9), gives the following result

$$
\hat{G}\left(X_{1}, X_{2}\right)=\sum_{j=1}^{4} \hat{G}_{j}\left(X_{1}, X_{2}\right),
$$


where

$$
\begin{aligned}
& \hat{G}_{1}\left(X_{1}, X_{2}\right)=\frac{1}{M_{-}^{4}} \int_{\Sigma} \int_{\Sigma} d \Sigma_{1}^{\mu} d \Sigma_{2}^{\nu} \hat{G}\left(x_{1}, x_{2}\right) \stackrel{\leftrightarrow}{\partial}_{1 \mu} \stackrel{\leftrightarrow}{\partial}_{2 \nu} F\left(x_{1}\right) F^{*}\left(x_{2}\right), \\
& \hat{G}_{2}\left(X_{1}, X_{2}\right)=\frac{1}{M_{-}^{4}} \int_{\Sigma} \int_{\Sigma} d \Sigma_{1}^{\mu} d \Sigma_{2}^{\nu}\left[\square_{1} \hat{G}\left(x_{1}, x_{2}\right)\right] \stackrel{\leftrightarrow}{\partial}_{1 \mu} \stackrel{\leftrightarrow}{\partial}_{2 \nu} f\left(x_{1}\right) F^{*}\left(x_{2}\right), \\
& \hat{G}_{3}\left(X_{1}, X_{2}\right)=\frac{1}{M_{-}^{4}} \int_{\Sigma} \int_{\Sigma} d \Sigma_{1}^{\mu} d \Sigma_{2}^{\nu}\left[\square_{2} \hat{G}\left(x_{1}, x_{2}\right)\right] \stackrel{\leftrightarrow}{\partial}_{1 \mu} \stackrel{\leftrightarrow}{\partial}_{2 \nu} F\left(x_{1}\right) f^{*}\left(x_{2}\right), \\
& \hat{G}_{4}\left(X_{1}, X_{2}\right)=\frac{1}{M_{-}^{4}} \int_{\Sigma} \int_{\Sigma} d \Sigma_{1}^{\mu} d \Sigma_{2}^{\nu}\left[\square_{1} \square_{2} \hat{G}\left(x_{1}, x_{2}\right)\right] \stackrel{\leftrightarrow}{\partial}_{1 \mu} \stackrel{\leftrightarrow}{\partial}_{2 \nu} f\left(x_{1}\right) f^{*}\left(x_{2}\right) .
\end{aligned}
$$

In these expressions $F(x) \equiv \square f(x)-M_{+}^{2} f(x)$. The function $f(x)$ must also satisfy the following 'initial conditions'

$$
\begin{aligned}
\left.f(x)\right|_{t=T} & =0, \\
\left.\partial_{t} f(x)\right|_{t=T} & =0, \\
\left.\square f(x)\right|_{t=T} & =0, \\
\left.\partial_{t} \square f(x)\right|_{t=T} & =M_{-}^{2} \delta^{3}(x),
\end{aligned}
$$

which play the role here of eq. (11) in the Klein-Gordon case.

Fearsome as it looks, this initial-value problem can be solved, and leads to functions, $f^{-}(x)$, which are basically identical with those that are found for the Klein-Gordon case. In particular, their support becomes infinitely small as $(T-t) \rightarrow \infty$, requiring a coefficient function that varies like $1 / \sigma\left(x_{1}, x_{2}\right)$ near the horizon. The new feature, though, is that the function that must be this singular involves not just $\hat{G}\left(x_{1}, x_{2}\right)$, but also its derivatives. It is these derivative terms that save the day: acting on $\hat{G}\left(x, x^{\prime}\right)$ they convert its $\log \sigma\left(x, x^{\prime}\right)$ behaviour into the $1 / \sigma\left(x, x^{\prime}\right)$ that is required for a nonzero result.

\section{Summary}

We have presented a derivation of the Hawking radiation within a simple model for which the ultraviolet regularization has been made explicit. This calculation permits the regularization-dependence of the Hawking flux to be explicitly displayed. It is found that the cutoff dependence is exponentially small in the limit that $\Lambda / T_{H} \gg 1$. Since the PauliVillars regularization used is slice-independent, this result agrees with what one would 
expect from the 'nice-slice' argument in favour of the irrelevance of the details of highenergy physics on the prediction of Hawking radiation.

The computation scheme of Fredenhagen and Haag [6] is used throughout, in which the Hawking radiation is directly related to the coincident singularity of the two-point function as both of its position arguments approach one another and the event horizon. We show that there is no contradiction in this approach between having a nonzero Hawking flux in the regulated theory, even though the resulting regulated two-point function is nonsingular in the coincidence limit.

\section{Acknowledgments}

We thank Ted Jacobson for explaining to us the mysteries of Ref. [6], and both Ted and Rob Myers for useful discussions. This research was partially funded by funds from the N.S.E.R.C. of Canada and les Fonds F.C.A.R. du Québec. 


\section{References}

[1] See, for example, S. Weinberg, Physica 96A (1979) 327; J. Polchinski, Nucl. Phys. B231 (1984) 269; J. Polchinski, in the proceedings of TASI 1992 (Boulder Colorado); J. F. Donoghue, Phys. Rev. D50 (1994) 3874.

[2] S.W. Hawking, Nature 248 (1974) 30; Comm. Math. Phys. 43 (1975) 199.

[3] W.G. Unruh, Phys. Rev. D14 (1976) 870, Phys. Rev. D15 (1977) 365.

[4] T. Jacobson, Phys. Rev. D44 (1991) 1731.

[5] T. Jacobson, Phys. Rev. D48 (1993) 728 .

[6] K. Fredenhagen and R. Haag, Comm. Math. Phys. 127 (1990) 273.

[7] D. A. Lowe, J. Polchinski, L. Susskind, L. Thorlacius and J. Uglum, Black Hole Complementarity vs. Locality, hep-th/9506138; J. Polchinski, "String Theory And Black Hole Complementarity", hep-th/9507094.

[8] P.B. Gilkey, J. Diff. Geom. 10 (1975) 601; S.M. Christensen, Phys. Rev. D14 (1976) 2490, B.S. DeWitt, Phys. Rep. C19 (1975) 296.

[9] S. Weinberg, Gravitation and Cosmology: Principles and Applications of the General Theory of Relativity (1982), (New York: Wiley). 